\title{
$\mathrm{SiC}$ 衬底上石墨烯的性质、改性及应用研究
}

\author{
方楠刘风刘小瑞廖瑞涃缪灵* 江建军 \\ (华中科技大学 光学与电子信息学院 武汉 430074)
}

\begin{abstract}
摘要 大面积高质量石墨烯的制备及其改性对于纳电子器件相关研究有重要意义. 本文综述了近年来 $\mathrm{SiC}$ 衬底上石墨 烯的相关研究，包括外延法制备石墨烯、石墨烯与 $\mathrm{SiC}$ 衬底的作用机理、 $\mathrm{SiC}$ 祄底上石墨烯的改性方法以及外延石墨 烯在器件等方面应用的重要进展. 目前, 外延法的工艺较为成熟且制备的较大面积石墨烯品质较好. SiC 衬底和石墨烯 之间的相互作用与祄底的表面原子种类、表面态、原子成键、钝化程度、电荷转移等密切相关，其对石墨烯的电子能 带、载流子种类产生明显影响. 实验与理论计算的结合可望加深对 $\mathrm{SiC}$ 衬底与石墨烯作用机理的理解, 并指导外延石 墨烯改性及其在器件应用方面的进一步研究.
\end{abstract}

关键词 石墨烯; 祄底; $\mathrm{SiC}$; 改性; 外延法

\section{Progress of Properties, Modification and Applications of Graphene on SiC Substrate}

\author{
Fang, Nan Liu, Feng Liu, Xiaorui Liao, Ruixian Miao, Ling* Jiang, Jianjun \\ (School of Optical and Electronic Information, Huazhong University of Science and Technology, Wuhan 430074, China)
}

\begin{abstract}
It is significant to prepare large-scale and high-quality graphene on SiC substrate for the research of the device in nanoelectronics. This review presents a brief overview on the progress in the growth mechanism for epitaxial graphene on $\mathrm{SiC}$ substrate surface, the methods of modification and the research of graphene-based devices. Graphene has attracted the interest of the scientists due to its exceptional electronic properties, which make it a promising material for nanoelectronics. Nowadays, the high-quality epitaxial graphene can be obtained with the smooth morphology, few defects, large size by controling the environment, annealing temperature and vapor pressure. The special structure that the first carbon layer grown above the Si-terminated $\mathrm{SiC}$ substrate form strong covalent bonds with substrate, results in some unusual properties such as opening a band gap in the graphene bilayer and a n-type doping of the graphene layers. The rotated fault, which is owed to decoupling effect, has been observed in C-terminated multilayer graphene. And such intrinsic properties of graphene depend on the atom type of SiC surface, surface state, weakness of bonds and the transformation of the electrons between the fewlayer graphene and $\mathrm{SiC}$ substrate. Some limitations (such as little band gap, intrinsic $n$-type character, etc.) resulted from the properties of epitaxial graphene must be overcome for the device application. Thus tremendous effort has been devoted to achieve the deliberate control of the band gap, the density and character of its charge carriers. Having a deep understanding of the unique properties for epitaxial graphene, this article then selectively reviews modification methods by considing the type of atoms, size of atoms, work function and electron affinity. In order to manufacture well-behaved devices, many of the fundamental problems of graphene-based eletronics, including methods to make metal contacts, nanoscale patterning and other technical details should be addressed perfectly. It is also important to control the material of gate, modulate the face of substrate, the width of channel in order to improve the performance of the graphene-based devices such as carrier mobility and $I_{\mathrm{on}} / I_{\mathrm{off}}$ ratios. The combination between the experiment and calculation (such as Monte Carlo simulation, density functional theory, molecular dynamics simulation etc.) can make a deeper understanding of the effects of SiC substrate and the methods of modification, which can be served as a guide for further research.
\end{abstract}

Keywords graphene; substract; $\mathrm{SiC}$; modification; epitaxy

\section{1 引言}

石墨烯自发现以后，由于其奇特优异的物理性质， 迅速成为国际研究热点 ${ }^{[1]}$. 石墨烯具有特殊的电子能带 结构, 其价带 $\pi$ 电子和导带 $\pi *$ 电子相交于费米能级 ${ }^{[2]}$. 能带的交叠点为狄拉克点, 此时表现出载流子具有线性 能量波矢色散关系、有效质量为零等有趣特性 ${ }^{[3]}$. 传统 半导体材料难以在 $30 \mathrm{~K}$ 以上观察到量子霍尔效应, 而
石墨烯在室温下即具有量子霍尔效应 ${ }^{[4,5]}$. 在当前大量 石墨烯制备、性质测试、器件应用等方面，石墨烯一般 均依附于祄底之上, 衬底对石墨烯的电学、光学性质等 影响较为明显. 通过外延法制备较大面积石墨烯的品质 较好, 且可直接应用于场效应管等器件而不用进行祄底 转移 ${ }^{[6]}$, 因此在微纳电子学研究中有光明的应用前景. 此制备方法得到的石墨烯有其特殊的结构与性质. $\mathrm{SiC}$ 祄底上的多层石墨烯存在屏蔽效应，能带结构与单层石

\footnotetext{
*E-mail: miaoling@hust.edu.cn

Received July 9, 2012; published September 12, 2012.
} 
墨烯较为相似. 外延石墨烯性质与 $\mathrm{Si}$ 和 $\mathrm{C}$ 之间的缓冲层 (即与衬底接触的第一层石墨烯)、石墨烯层与层之间的 耦合关系有关. $\mathrm{SiC}$ 衬底上的石墨烯在不加以修饰的情 况下, 能表现出 $\mathrm{N}$ 型掺杂的特性, 能带轻微打开. 如何 通过改性, 以使外延法制备的石墨烯具有特定电子特性 以满足器件需要是当前研究热点 ${ }^{[7]}$. 本文综述了近年来 $\mathrm{SiC}$ 祄底上石墨烯的相关研究, 包括外延法制备石墨 烯、石墨烯与 $\mathrm{SiC}$ 衬底的作用机理、 $\mathrm{SiC}$ 衬底上石墨烯 的改性方法以及外延石墨烯在器件等方面应用的重要 进展.

\section{2 石墨烯的制备方法}

相比于微机械分离法 ${ }^{[8]}$ 、化学溶剂法 ${ }^{[9]}$ 和脉冲激光 沉积法 ${ }^{[10]}$, 现在研究较多的石墨烯生长方法是 $\mathrm{SiC}$ 衬底 上外延生长法 ${ }^{[11]}$ 和化学气相沉积法(CVD) ${ }^{[12,13]}$. CVD 方 法可以制备出大面积、高质量的石墨烯, 但是对于祄底, 尤其是起催化作用的金属祄底有一定的依赖性. 在应用 于微纳电子学研究与应用时, CVD 法制备的石墨烯需要 进行祄底转移, 这增加了工艺的复杂性 ${ }^{[14,15]}$. 而外延法 制备石墨烯中多采用 $\mathrm{SiC}$ 半导体衬底, 此祄底可直接应 用于场效应管等器件的制造, 无需额外的祄底转移等过 程. 但是外延石墨烯对 $\mathrm{SiC}$ 祄底表面缺陷较为敏感, 从 而对载流子的迁移率有一定影响. 当前对于外延法制备 石墨烯工艺的研究多关注于如何通过控制生长温度、生 长速率等因素以使得外延法生长的石墨烯层数较为一 致且迁移率较高.

\section{1 外延法生长石墨烯的工艺发展}

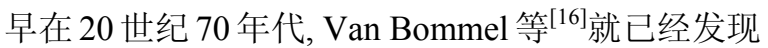
可以通过加热的方式使 SiC (0001)表面石墨化的现象. 90 年代, 通过应用 STM 等实验手段观察, 发现外延法 所得到的石墨片层电子输运性质优异 ${ }^{[17,18]}$. 在 2004 年, Berger 等 ${ }^{[19]}$ 在该法制备的石墨片层中观察到了量子霍 尔效应, 并且预见外延石墨烯能够应用到纳米器件中. $\mathrm{SiC}$ 祄底上外延生长石墨烯的基本工艺如下：在超高真 空环境和高温下, 通过加热 $\mathrm{SiC}$ 祄底, 使其中的 $\mathrm{Si}$ 原子 升华, 留下的富 $\mathrm{C}$ 原子表面则石墨化, 重构形成石墨烯 层. 早期外延法生长制备石墨烯, 普遍采用超真空环境 (UHV). 2007 年, De Heer 等 ${ }^{[20]}$ 通过外延法制备出平均厚 度少至一层的较为理想的石墨烯. 制备所采用的 $\mathrm{SiC}$ 多 是 $4 \mathrm{H}$ 和 $6 \mathrm{H}$ 结构. 同时 $\mathrm{Si}$ 原子升华的面也可以有 $\mathrm{SiC}$ (0001) (以 $\mathrm{Si}$ 为衬底最后一层面)和 $\mathrm{SiC}(000 \overline{1}$ ) (以 $\mathrm{C}$ 为 衬底最后一层面)两种情况.

但是与 CVD 法制备的石墨烯相比, 在传统的 UHV 和高温环境下外延生长石墨烯的品质并不算好，会产生 层数不一的石墨烯岛状结构. 这是由于 $\mathrm{C}$ 原子的扩散、 重构并不完全所致. 升高温度可使 $\mathrm{C}$ 原子重构趋于完 全, 但同时会使得 $\mathrm{Si}$ 原子的升华速率加快, 导致了更多 需要重构的 $\mathrm{C}$ 原子的产生, 当重构不完全时, 即产生了
岛状结构. 即制备的石墨烯生长速率(主要决定于 $\mathrm{Si}$ 原 子的升华速率)、品质同时与生长温度相关联. 在保证生 长速率的同时，很难通过改变实验条件，改善石墨烯的 品质 ${ }^{[21,22]} . \mathrm{Si}$ 原子有较高的升华速率时，表面石墨烯的 重构时间减少、品质下降 ${ }^{[23]}$. 为了克服在 UHV 环境中 生长速率仅与温度有关的缺点, 通过热平衡理论与反应 平衡理论的分析, 可以在腔内通入惰性气体、气态 $\mathrm{Si}$ 或乙硅烷等气体, 使得石墨烯生长速率减慢. CCS (confinement controlled sublimation)方法采用了反应炉 (furnace)这一在传统 CVD 方法中使用较多的半密闭体 腔. 此时气体环境、反应温度均对石墨烯的品质、层数 有较大影响 ${ }^{[24]}$.

2011 年, Norimatsu 等 ${ }^{[25]}$ 通过在高压 $\mathrm{Ar}$ 气体环境中 观察石墨烯外延生长的物理过程, 强调了 $\mathrm{Si}$ 升华速率 在石墨烯重构过程中的决定性作用. 如图 1, De Heer 等 ${ }^{[26]}$ 在气态 $\mathrm{Si}$ 环境下通过调节体腔的孔径和环境气压 实现了在 $1600{ }^{\circ} \mathrm{C}$ 反应温度下生长速率的调节. 从而在 生长速率适当的情况下, 制备出较高品质的石墨烯. Dass 等 ${ }^{[27]}$ 比较了 $\mathrm{Ar}$ 惰性气体环境与气态 $\mathrm{Si}$ 环境对于石 墨烯品质的影响. Si 终端面上生长的石墨烯在气态 $\mathrm{Si}$ 环 境下比在 $\mathrm{Ar}$ 惰性气态环境下有更好的品质. 需要指出 的是, 为了防止热泳现象的发生, 减小温度梯度是必要 的. Srivastava 等 ${ }^{[28]}$ 指出由于氧化作用, $\mathrm{C}$ 终端面上生长 的石墨烯在 $\mathrm{Ar}$ 环境中生长时会产生异质结构. 在乙硅 烷环境下生长石墨烯则可以较好地避免石墨烯氧化.

用 CCS 法制备石墨烯时，通过适当升高温度，可使 石墨烯层数增加 ${ }^{[29]}$. Vecchio 等 ${ }^{[30]}$ 通过 AFM, HR-XTEM 等手段观察了外延石墨烯的微观结构, 认为由于祄底与 石墨烯的热膨胀系数不同, 反应温度过高, 冷却时界面 所产生的不匹配现象较为明显, 将会导致石墨烯褶皱产 生.

\section{Vacuum or inert gas}

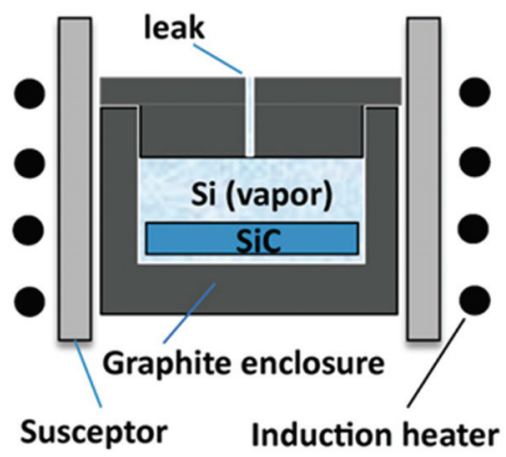

图 1 反应炉中生长石墨烯的方法图示 ${ }^{[26]}$

Figure 1 The image of CCS method of obtaining epitaxial graphene in furnace $^{[26]}$

\section{$2.2 \mathrm{SiC}$ 衬底上外延生长石墨烯的结构}

外延法生长石墨烯时, 第一层石墨烯通常覆盖整 个衬底表面，第二层石墨烯由第一层石墨烯下方的 $\mathrm{Si}$ 
原子升华后剩余的 C 原子重构生成, 而多层石墨烯仅仅 在部分区域生长, 形成岛状结构. 当缺陷较多、晶粒较 小时, $\mathrm{Si}$ 原子可通过缺陷和边界升华, 导致石墨烯层数 增多. 多层石墨烯会阻碍祄底内部 Si 原子的升华, 因此 外延法制备所得石墨烯多是 1 3 层. 由于在石墨烯中 C 原子密度 $\left(3.82 \times 10^{15} \mathrm{~cm}^{-2}\right)$ 大约是 $\mathrm{SiC}$ 中 $\mathrm{C}$ 原子密度 $\left(1.22 \times 10^{15} \mathrm{~cm}^{-2}\right)$ 的 3 倍, 生长一层石墨烯大约需要升 华三层 $\mathrm{SiC}$ 中的 $\mathrm{Si}$ 原子. Hass 小组 ${ }^{[31]}$ 通过 XPS 分析, 深 入讨论了界面处的晶格常数, 提出了存在表面褶皱的微 观解释, 得到了较为准确的 $\mathrm{SiC}$ 祄底上石墨烯的结构模 型. 如图 $2^{[32]}$, 在祄底和石墨烯的界面处, 石墨烯层不 是简单地位于祄底之上, 而是更加复杂的空间构型, 且 界面复杂的构型延伸到了祄底之中.

界面具体生长方式的探究, 为制备过称中所产生的 岛状结构与线性断层(threading screw dislocation, TSD) 提供了理论依据 ${ }^{[33 \sim 35]}$. Hite 等 ${ }^{[34]}$ 以 TSD 为出发点, 探究 了外延法步骤中, 石墨烯的具体生长过程. 他们观察 到移除石墨烯后 TSD 依旧存在这一现象, 判断 TSD 是 在石墨烯生长之初产生的, 并且石墨烯更倾向于生长在 TSD 附近区域. 所以, TSD 在 $\mathrm{Si}$ 升华过程中起到了非 常重要的作用.
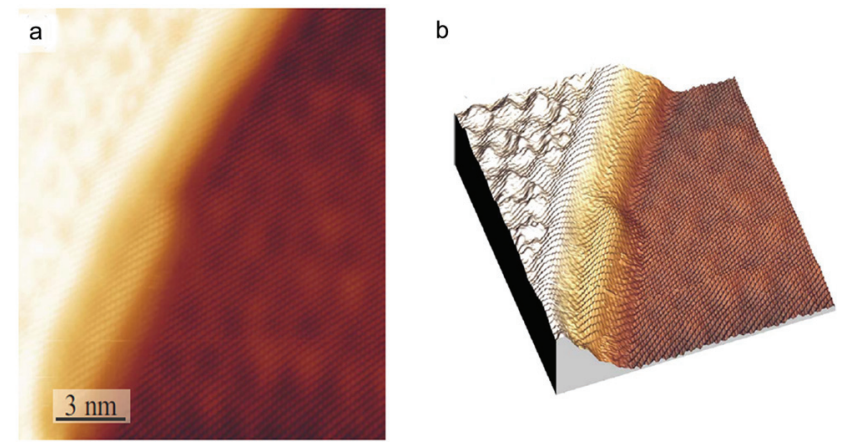

图 2 (a) 石墨烯台阶的 STM 微观形貌图和(b) 3D 模拟图 ${ }^{[32]}$

Figure 2 (a) STM image of a graphene-covered SiC step and (b) 3D visualization of the image ${ }^{[32]}$

\section{$3 \mathrm{SiC}$ 衬底外延生长石墨烯的性质}

$\mathrm{SiC}$ 祄底与石墨烯的晶格匹配较好, 两者之间有较 强的相互作用. $\mathrm{SiC}$ 半导体表面的功函数、界面态强弱与 钝化程度决定了与半金属性石墨烯之间的肖特基势垒, 从而影响了石墨烯的掺杂程度及能带结构等性质. 外延 生长所得多层石墨烯的性质类似于单层石墨烯 ${ }^{[2,36]}$. 通 过计算和实验得到在 $\mathrm{SiC}$ 衬底上生长的石墨烯性质主要 由过渡层(祄底上面 1 2 层) 决定 ${ }^{[37]}$, 而之上的石墨烯对 其影响很小 ${ }^{[38]}$.

$\mathrm{SiC}$ 祄底对石墨烯的结构影响 ${ }^{[39]}$, 主要体现在以下 几个方面. 一是缓冲层结构 (多称为第 0 层), 即与 $\mathrm{SiC}$ 祄 底有强烈作用的第一层 $\mathrm{C}$ 原子层的形成 ${ }^{[40]}$; 二是缓冲层 之上的石墨烯层间耦合作用不同于一般多层石墨烯, 导
致了特殊的多层角度旋转结构. 过渡层结构分为 2 种模 型, 即 $\mathrm{SiC}$ (0001) ( $\mathrm{Si}$ 终端面)和 $\mathrm{SiC}(0001)$ (C 终端面). 不同结构的缓冲层所表现性质不同, 且石墨烯层数较多 时性质较为独特, 下面将分别介绍.

\section{1 在 SiC (0001)面的缓冲层性质}

在 $\mathrm{SiC}(0001)$ 面的外延石墨烯和补底之间有很强的 耦合作用, 会使石墨烯的能带结构发生较大改变 ${ }^{[41]}$. 具体表现为会使石墨烯具有 $\mathrm{N}$ 型掺杂的特性, 同时轻微 地打开能带带隙. 图 3 中是以 $\mathrm{SiC}$ 祄底中 $\mathrm{Si}$ 原子为祄底 表面层的模型 ${ }^{[42]}$. 图 4 是利用第一性原理计算所得的对 应能带图. 部分 $\mathrm{Si}$ 原子和之上的 $\mathrm{C}$ 原子形成了强烈的共 价键，没有与石墨烯成键的 $\mathrm{Si}$ 原子则是孤立原子，导致 了悬挂键的产生, 呈现出 $\mathrm{N}$ 型半导体特性. 低能电子衍 射(LEED)等实验数据则表明, 由于 $\mathrm{Si}$ 原子会和其上的 石墨烯层产生共价作用使得石墨烯中离域性较强的 $\pi$ 键 能级降低, 能带打开, 而缓冲层之上的石墨烯又恢复了 通常悬浮石墨烯的性质, 狄拉克点重新出现 ${ }^{[41,43]}$. 这些 性质为之后的掺杂改性研究提供了理论基础 ${ }^{[44]}$. 值得 注意的是, 在此面上生长的石墨烯与祄底化学作用较为 强烈，导致散射作用较强，从而使得载流子迁移率较低. Hibino 等 ${ }^{[45]}$ 利用 LEEM 等手段观测当温度升高时, 由于 载流子浓度升高, 迁移率会降低. 他们认为这主要是点 缺陷、祄底的错位缺陷等造成的.
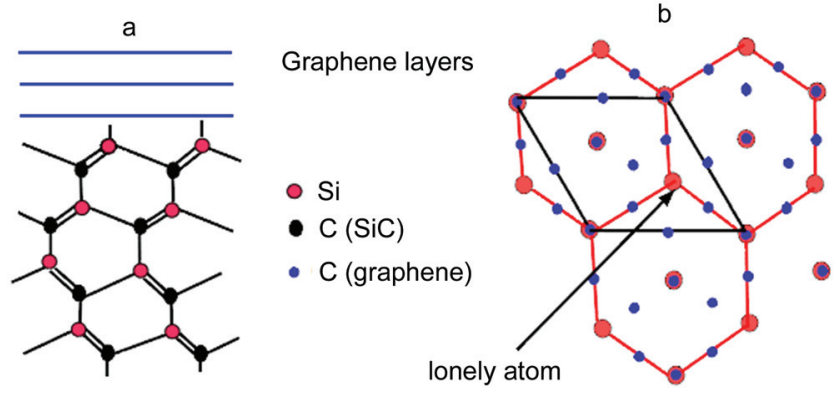

图 $3 \mathrm{Si}$ 终端面上外延石墨烯的缓冲层结构示意图 ${ }^{[42]}$. a: 侧视图; b: 俯视图

Figure 3 Interface geometry of the cell for a Si-terminated $\mathrm{SiC}$ face ${ }^{[42]}$. a: side view; b: top view

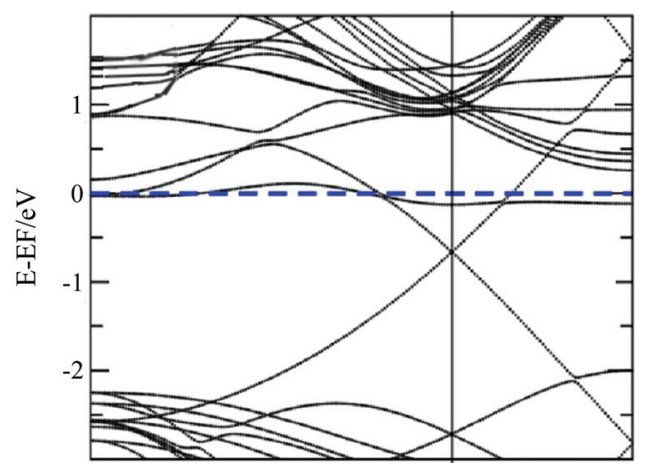

图 4 以 $\mathrm{Si}$ 为衬底表面, 在缓冲层之上生长单层石墨烯的能带图 ${ }^{[42]}$ Figure 4 Dispersion curves for $2 \mathrm{C}$ layers on top of the Si-terminated surface $^{[42]}$ 


\section{2 在 $\mathrm{SiC}(000 \overline{1})$ 面的缓冲层性质}

Hass 小组依据第一性原理计算分析了 $\mathrm{SiC}(000 \overline{1})$ 面 上外延石墨烯的能带结构 ${ }^{[44]}$. 如图 5, SiC (0001) 面表现 的性质与 $\mathrm{SiC}(0001)$ 面表现的性质有很大的不同. 一是 石墨烯特有的圆雉形能带结构并没有消失, 即能带基本 没有打开; 二是费米面基本没有移动, 不显示 $\mathrm{N}$ 型半导 体的特性; 三是石墨烯保留了原有的很高迁移率. $\mathrm{Wu}$ 等 ${ }^{[46]}$ 通过实验，观察到在 $300 \mathrm{~K}$ 下，此结构中石墨烯的 迁移率达到 $15000 \mathrm{~cm}^{2} /(\mathrm{V} \cdot \mathrm{s})$, 并且验证了上述性质. 这 些性质与石墨烯层和衬底相对较弱的耦合作用有关. Tang 等 ${ }^{[47]}$ 通过原子力显微镜(AFM)、激光拉曼光谱 (Raman)等实验技术证实 $\mathrm{C}$ 终端上的外延石墨烯有较多 $\mathrm{sp}^{2}$ 杂化形式的 $\mathrm{C}$ 原子. 在此面外延生长的石墨烯较 $\mathrm{SiC}(0001)$ 面有相对较大的面积, 方便于器件方面的应 用 ${ }^{[48]}$.

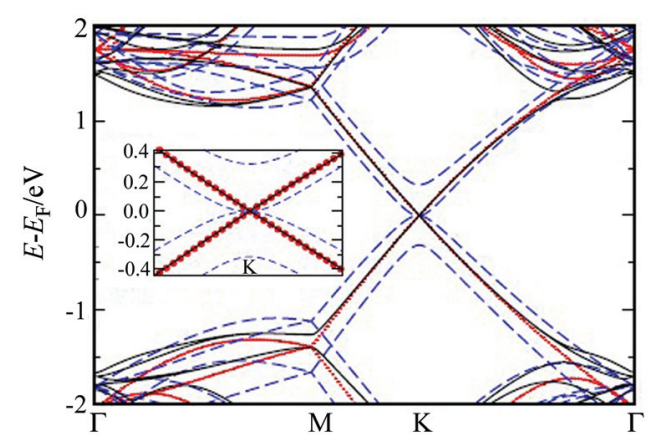

图 5 蓝色虚线为以 $\mathrm{AB}$ 方式堆叠(以 $\mathrm{Si}$ 为祄底表面)的石墨烯层能带 图; 黑色实线为考虑了旋转缺陷的双层石墨烯能带图(以 $\mathrm{C}$ 为祄底表 面); 红色点图为单层悬浮石墨烯的能带图 ${ }^{[44]}$; 插图为在 $\mathrm{K}$ 点附近的 放大图

Figure 5 Calculated band structure for three forms of graphene. Isolated graphene sheet (dots), $\mathrm{AB} \cdots$ graphene bi-layer that is Si-terminated (dashed line), and C-terminated bi-layer graphene (solid line). Inset shows details of band structure at the K-point ${ }^{[44]}$

\section{3 多层外延石墨烯的性质}

由于祄底和缓冲层之间的作用, 以 $\mathrm{C}$ 原子层为祄底 表面的多层石墨烯表现出悬浮石墨烯(freestanding graphene)的性质, 具有很高的载流子迁移率, 很短的弛豫 时间 ${ }^{[49 ~ 51]}$. 本文着重介绍当前研究较为透彻的两点性 质, 一是角度无序性, 即在 C 终端面上的外延石墨烯中 层与层之间旋转角不固定; 二是屏蔽效应, 即当祄底掺 杂或加偏置电压时, 不同层的石墨烯载流子浓度变化不 同，上层石墨烯的载流子浓度变化很小. 关于费米速率 减少、范霍夫奇点等问题 ${ }^{[52]}$, 目前理论和实验数据没有 很好的统一, 有待进一步的研究.

\subsection{1 多层石, 墨烯的角度无序性}

$\mathrm{Si}$ 终端面上的石墨烯一般以 $\mathrm{AB}$ 型方式堆叠, 而 $\mathrm{C}$ 终端面上的石墨烯具有角度无序性, 其堆叠方式不同于 一般的 $\mathrm{AB}$ 或者 $\mathrm{AC}$ 型, 而是比较复杂的形式 ${ }^{[53 \sim 55]}$. 第 一性原理计算 ${ }^{[56]}$ 和实验手段, 如 APRES 拉曼光谱 ${ }^{[57]}$ 和 $\mathrm{STM}^{[58]}$ 均验证了这一情况. $\mathrm{SiC}$ 衬底上的多层石墨烯与
悬浮多层石墨烯不同，其能带结构不简并，在低能级处 能级分裂，因此不同层数的石墨烯能够被分辨 ${ }^{[59]}$. 由于 旋转缺陷在层与层之间相关联，因此在较多层数的石墨 烯中，其角度无序性并不高 ${ }^{[60]}$.

Hass 等 ${ }^{[61]}$ 指出不同于一般的石墨烯, 如图 6 所示, $\mathrm{SiC}$ 衬底 $\mathrm{C}$ 终端面上的石墨烯结构由 $\sqrt{13} \times \sqrt{13}$ 有旋转 缺陷的晶胞组成, 旋转角为 $46.1^{\circ}$. 他们认为缓冲层在这 一现象中起到了重要作用. Hiebel 小组 ${ }^{[62]}$ 则通过加偏置 电压观察了第一层石墨烯与缓冲层之间的耦合与角度 关系. 相比于缓冲层与祄底的耦合强度，第一层石墨烯 与缓冲层的耦合强度很弱, 不足以固定旋转角. 第一层 石墨烯的旋转角在 $14^{\circ}$ 到 $22^{\circ}$ 之间 ${ }^{[63]}$. Magaud 等 ${ }^{[64]}$ 通过 第一性原理的计算, 利用多层无序性对石墨烯整体能量 的贡献大小解释了其角度无序性. 当改变石墨烯层与层 之间的耦合角度时，其整体能量差别不超过 $15 \mathrm{meV}$. 从而很好地解释了石墨烯层与衬底之间弱的耦合作用. 需要指出的是, 这种角度的无序性, 不存在于其他制备 方法所得石墨烯中. 值得一提的，角度无序性可导致在 C 表面外延生长的石墨烯有很强的线性磁致电阻 ${ }^{[33]}$. Friedman 小组 ${ }^{[65]}$ 通过实验验证了温度对磁致电阻的影 响.
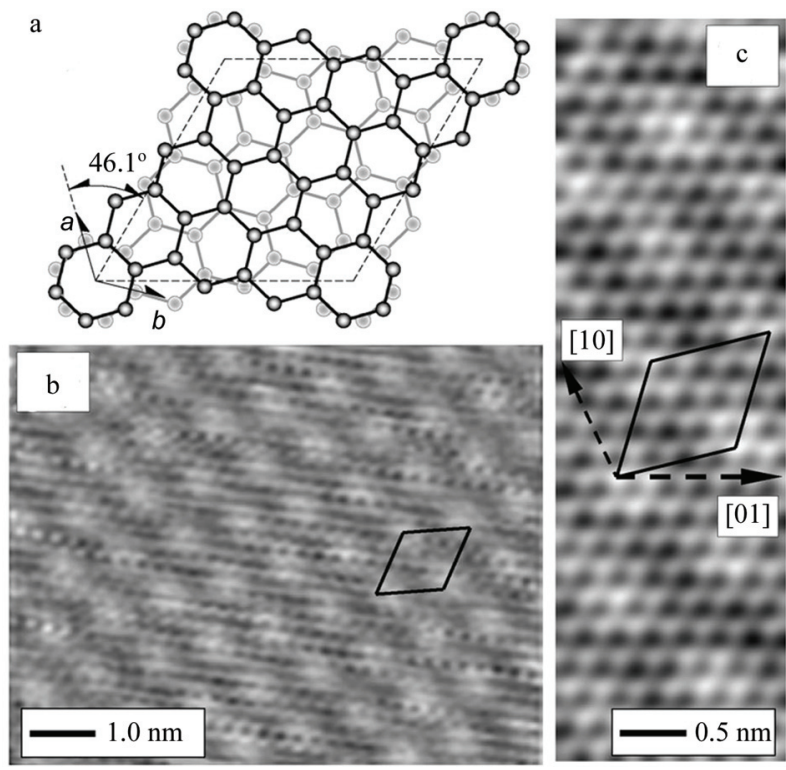

图 6 (a) 考虑旋转缺陷所建的 C 终端面上石墨烯模型图; (b) STM 晶 胞图; (c) 高分辨 STM 图 ${ }^{[44]}$

Figure 6 (a) Schematic of C-terminated mutilayer graphene considered with order fault; (b) STM image of C-face graphene showing a periodic superlattice with a $\sqrt{13} \times \sqrt{13}$ cell; (c) High resolution STM image ${ }^{[44]}$

\subsection{2 多层石墨烯的屏蔽效应}

C 终端面上生长的石墨烯层与层之间弱的耦合作 用，使得每层表现得如同悬浮石墨烯一般，这种特性阻 止了石墨烯的掺杂效应和能带结构改变. 另外衬底表面 孤立 C 原子会产生表面态. 当表面态较大时，衬底与表 
面态之间产生势垒. 根据金半接触理论，从衬底流向石 墨烯的电子主要由表面态提供, 从而屏蔽了衬底内部费 米势的改变对石墨烯载流子浓度的影响.

早在 2007 年, Berger 等 ${ }^{[39]}$ 通过第一性原理计算定性 指出了载流子浓度仅在第一层改变很大，在上层石墨烯 则改变很小. 2009 年, Sprinkle 等 ${ }^{[66]}$ 通过 ARPES 对 $\mathrm{SiC}$ 祄底 C 终端面上多层石墨烯的能带进行了分析, 认为石 墨烯层与祄底表面之间的共价作用使上层石墨烯的性 质主要由缓冲层决定, 而祄底掺杂浓度的影响程度很 小. 2010 年, Sun 等 ${ }^{[67]}$ 通过百浦探测(pump-probe)实验定 量研究了在多层石墨烯样品不同片层中电子浓度的差 异. 石墨烯第一层的掺杂程度最大, 之上的 3 层掺杂程 度依次减小, 费米能级从第一层到第四层分别为狄拉克 点之上的 360, 215, 140 和 $93 \mathrm{meV}$, 图 7 显示了不同层之 间电子浓度的差异. 电子浓度的改变仅仅在界面附近少 数几层有所体现, 其上的石墨烯层电子浓度几乎不变, 显示出屏蔽效应.

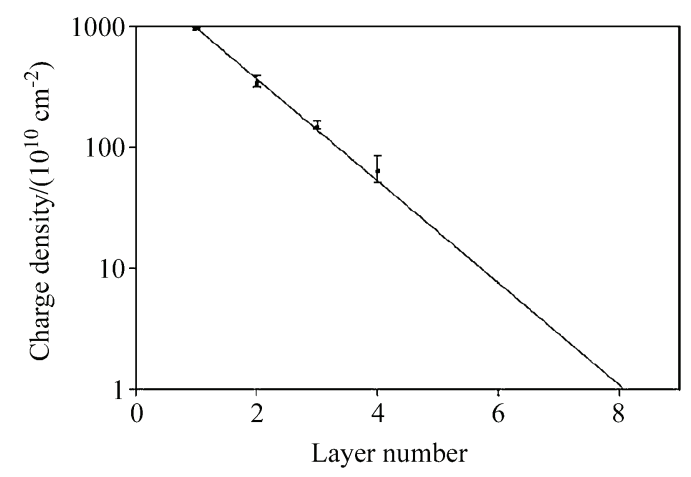

图 $7 \mathrm{SiC}$ 祄底上石墨烯的电荷密度与层数变化关系示意图 ${ }^{[67]}$

Figure 7 Exponential fit to the experimental charge density of the first four heavily doped layers ${ }^{[67]}$

2011 年, Huang 等 ${ }^{[68]}$ 通过第一性原理的计算, 探究 了屏蔽效应的成因. 当采用 $\mathrm{B} 、 \mathrm{P}$ 原子在缓冲层附近进 行掺杂改性时, 上层石墨烯的载流子浓度仅仅轻微改 变. 在进行 $\mathrm{N}$ 型或 $\mathrm{P}$ 型掺杂改性时, 费米面附近的局域 界面态限制了上层石墨烯的掺杂效应. $\mathrm{SiC}$ 衬底 $\mathrm{C}$ 终端 面上生长的多层石墨烯不易受祄底缺陷的影响, 具有单 层石墨烯的高迁移率等特性.

\section{4 对 SiC 衬底上石墨烯的改性}

为使制备得到的石墨烯更适合于各种微纳器件的 应用, 需要对其进行改性 ${ }^{[69]}$. 如适当打开能带带隙能保 证器件在关断时, 热效应等产生的漏电流较小, 从而具 有良好的关断特性, 利于逻辑器件性能的提高. 打开能 隙的方法主要有如下两种，一是利用量子限域效应，将 石墨烯由二维平面结构裁剪成一维线性结构, 可对其电 子结构产生明显影响. 但受到纳米量级刻蚀工艺的限 制，使得石墨烯的载流子迁移率下降、响应时间变长， 在应用上有一定局限性 ${ }^{[70]}$. 二是利用化学改性方法改
变 $\mathrm{C}-\mathrm{C}$ 键的杂化方式, 使之从 $\mathrm{sp}^{2}$ 杂化转变为 $\mathrm{sp}^{3}$ 杂化, 从而改变能带结构 ${ }^{[71,72]}$. 此外, 传统的集成电路工艺是 通过掺杂、离子注入等方式进行 $\mathrm{N}$ 型, $\mathrm{P}$ 型性质的转变, 以实现 p-n 结、MOS 等结构的制造. 由于石墨烯的化学 结构异常稳固, 传统工艺难以实现对石墨烯的掺杂改 性，且 $\mathrm{SiC}$ 衬底上外延制备的石墨烯一般表现 $\mathrm{N}$ 型掺杂 的特性, 所以如何在保证较小的杂质散射、较大的载流 子迁移率同时, 对外延石墨烯进行 P 型改性在微纳器件 的应用方面具有重要意义 ${ }^{[44]}$.

\section{1 金属原子对 SiC 衬底上石墨烯的改性}

研究人员对金属衬底如 $\mathrm{Au}, \mathrm{Ni}, \mathrm{Ir}, \mathrm{Ru}, \mathrm{Rh}, \mathrm{Co}, \mathrm{Pt}$ 等 对石墨烯的影响, 已经有了较为深入的探索 ${ }^{[73]}$. 然而 通过金属原子修饰对 $\mathrm{SiC}$ 衬底上石墨烯进行改性的研究 不多，仍需要更为深入的理论和实验研究. 金属原子与 石墨烯的作用机理在研究其对 $\mathrm{SiC}$ 衬底上石墨烯的改性 作用中具有指导意义.

\subsection{1金属原子与石, 墨烯的作用机理}

金属原子与石墨烯之间的作用主要分为两种，一种 是强的化学相互作用, 如 $\mathrm{Co}, \mathrm{Ni}, \mathrm{Pd}$, 会破坏石墨烯的 $\pi$ 键结构; 另一种是吸附作用, 如 $\mathrm{Al}, \mathrm{Cu}, \mathrm{Ag}, \mathrm{Au}, \mathrm{Pt}$, 会 保留狄拉克点. 具体表现在吸附金属原子与石墨烯层之 间的距离差别上. 当距离较近时，化学作用较强，距离 较远时, 化学作用则较弱.

2008 年, Giovannett 等 ${ }^{[74]}$ 指出，当金属衬底与石墨 烯接触时, 功函数起到很重要的作用. 当金属的功函数 大于石墨烯的功函数时, 金属衬底可使石墨烯显示 $\mathrm{P}$ 型 半导体的特性. 因此 $\mathrm{Au}, \mathrm{Re}, \mathrm{Ir}, \mathrm{Pt}, \mathrm{Ge}$ 等功函数大于石 墨烯功函数的金属原子可作为 $\mathrm{P}$ 型改性的参考. Gierz 等 ${ }^{[75]}$ 通过将 $\mathrm{Bi}, \mathrm{Sb}$ 原子沉积在石墨烯层之上实现了狄 拉克点向费米能级的靠近. 但是随着沉积量的加大，不 能达到 $\mathrm{P}$ 型掺杂的目的，仅仅是减弱了 $\mathrm{N}$ 型掺杂的程度. 他们指出这种现象的产生是由于 $\mathrm{Bi}, \mathrm{Sb}$ 原子的电子功函 数不够大, 不能进一步引起电子从石墨烯到改性原子的 转移. Pletikosić 等 ${ }^{[76]}$ 通过对 $\mathrm{Ni}$ 衬底上的石墨烯进行 ARPES 分析, 观测到了石墨烯能带打开的现象.

Preobrajenski 等 ${ }^{[77]}$ 指出强烈的化学作用和晶格失 配，会导致表面褶皱的产生. Moritz 等 ${ }^{[78]}$ 则通过 LEED 等实验手段, 观测到 $\mathrm{Ru}$ 祄底和石墨烯层之间的距离不 一致，证明产生了较明显的褶皱，验证了上述观点.

\subsection{2 $\mathrm{Au}, \mathrm{Al}$ 原子对 $\mathrm{SiC}$ 衬底上石, 墨烯的改性}

Huang 等 ${ }^{[68]}$ 通过第一性原理的计算, 说明了 $\mathrm{Al}$ 对 $\mathrm{SiC}$ 祄底上石墨烯 $\mathrm{P}$ 型改性的可行性. Al 易于取代祄底 表面 $\mathrm{Si}$, 从而达到掺杂改性的目的. 当 $\mathrm{Al}$ 在祄底体内掺 杂时, 由于屏蔽效应，掺杂效果并不明显. Al 相较于 $\mathrm{Si}$, 有较低的电负性和较大的共价半径. 当取代祄底表面 $\mathrm{Si}$ 时, 可以与 $\mathrm{C}$ 之间形成强烈的化学键. 相比于直接在石 墨烯中进行掺杂，这种间接的掺杂方式在工艺上更易于 实现，并且可以使掺杂剂与导电沟道相隔离，减少散射， 
因此迁移率不会有明显的改变 ${ }^{[79]}$.

$\mathrm{Au}$ 对于 $\mathrm{SiC}$ 衬底上石墨烯的改性已经在实验条件 下得到证实. 2008 年, Varykhalov 等 ${ }^{[80}$ 通过在 $\mathrm{Ni}$ 衬底和 石墨烯之间插入 $\mathrm{Au}$ 的方式, 使石墨烯能带结构中的狄 拉克点重新出现. 费米面和狄拉克点重合, 说明了石墨 烯表现出类似未掺杂特性. Gierz 等先后于 2008 年 ${ }^{[75]}$ 和 2010 年 ${ }^{[81]}$ 通过在 $\mathrm{SiC}$ 祄底上外延生长的石墨烯上沉积 $\mathrm{Au}$ 的方法, 证实了 $\mathrm{Au}$ 对于 $\mathrm{SiC}$ 衬底上石墨烯的改性作 用. 利用石英振荡器激光校准, 可以较为精确地控制 $\mathrm{Au}$ 覆盖量. 由于只有在 $1000{ }^{\circ} \mathrm{C}$ 以上时, $\mathrm{SiC}$ 祄底升华 $\mathrm{Si}$ 、生成石墨烯的过程才容易发生. 因此 $\mathrm{Au}$ 在室温沉积 以后, 在 $800{ }^{\circ} \mathrm{C}$ 低温淬火不会生成多余的石墨烯片层. 他们利用 AFM, LEED, ARPES 等实验手段观测到, 当 在石墨烯上沉积 $\mathrm{Au}$ 时, $\mathrm{Au}$ 首先会插入到石墨烯与衬底 之间, 与祄底产生强烈的化学作用, 从而屏蔽了衬底对 石墨烯的影响, 使得缓冲层石墨烯恢复为有狄拉克点的 能带结构. 当覆盖的 $\mathrm{Au}$ 较多时, $\mathrm{Au}$ 会以团族的形式附 着在石墨烯表面. 通过进一步控制 $\mathrm{Au}$ 在石墨烯与衬底 之间的覆盖量, 可以达到 $\mathrm{N}$ 型或者 $\mathrm{P}$ 型掺杂的目的. 无 $\mathrm{Au}$ 修饰时, 单层石墨烯与衬底强烈的相互作用, 导致 了狄拉克点的消失 (图 8a). 控制反应温度可得层数较为 一致的双层石墨烯, 其能带结构与悬浮石墨烯相似, 即 狄拉克点出现(图 8b). 当 $\mathrm{Au}$ 覆盖 $1 / 3$ 的祄底时, 显示 $P$ 型掺杂的特性(图 $8 \mathrm{c}$ ). 当 $\mathrm{Au}$ 全部覆盖衬底的时候, 显示 $\mathrm{N}$ 型掺杂的特性(图 8d). 他们认为产生此现象的原因是 过大的 $\mathrm{Au}$ 覆盖量使得 $\mathrm{Au}$ 与石墨烯之间的距离增大, 从 而导致它们之间的化学作用减弱, 显示出 $\mathrm{P}$ 型掺杂的效 应，载流子浓度减小.

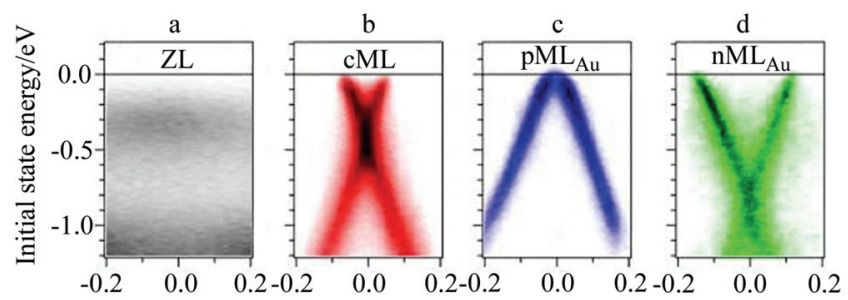

图 8 用 ARPES 得到的能带结构图. (a) 单层石墨烯的能带图; (b) 双 层石墨烯能带图; (c) Au 掺杂 P 型石墨烯能带图; (d) Au 掺杂 $\mathrm{N}$ 型石墨 烯能带图 ${ }^{[81]}$

Figure 8 Comparison of ARPES data for conventional graphene on $\mathrm{SiC}$ and graphene intercalated with $\mathrm{Au}$; (a) monolayer on the $\mathrm{SiC}$ substrate (b) bilayer graphene on the substrate; (c) the p-doped bilayer graphene; (d) the $\mathrm{n}$-doped bilayer graphene ${ }^{[81]}$

\section{2 非金属原子对 SiC 衬底上石墨烯的改性}

非金属原子对 $\mathrm{SiC}$ 衬底上石墨烯的改性主要通过表 面修饰如掺杂、钝化悬挂键、与祄底去耦合等方式 ${ }^{[82,83]}$. 由于石墨烯中的 $\mathrm{C}-\mathrm{C}$ 共价键很稳固, 所以修饰一般发 生在缓冲层和祄底之中. Huang 等 ${ }^{[68]}$ 通过第一性原理计 算分析了在 $\mathrm{SiC}$ 衬底 $\mathrm{C}$ 终端面上的石墨烯, 用 $\mathrm{B}, \mathrm{P}$ 等传 统半导体掺杂剂进行掺杂改性的可行性. 如图 9 的 b, c
中, B, P 对石墨烯分别起到 $\mathrm{P}$ 型, $\mathrm{N}$ 型掺杂的作用. 虽然 $\mathrm{B}, \mathrm{P}$ 原子的 $\mathrm{sp}^{2}$ 杂化半径比 $\mathrm{C}$ 大, 但由于缓冲层有较好 的应力伸缩性质，因此 $\mathrm{B}, \mathrm{P}$ 原子易于取代缓冲层的 $\mathrm{C}$ 原 子.

$\mathrm{H}$ 原子改性是通过针化悬挂键来与衬底去耦合 ${ }^{[84]}$. Balog 等 ${ }^{[85}$ 通过 STM 观察了石墨烯表面吸附 H 时的团 簇现象. Huang 等 ${ }^{[68]}$ 从理论上说明了钝化的改性作用. 如图 10 中 $\mathrm{d}, \mathrm{e}, \mathrm{f}$, 钝化以后, 悬挂键被 $\mathrm{H}$ 原子饱和, 表 面能级消失. 当表面态被 $\mathrm{H}$ 占据以后，屏蔽效应则会减 弱，因此进行 $\mathrm{N}$ 型, $\mathrm{P}$ 型掺杂时，载流子可以从衬底传递 到石墨烯, 掺杂效果增强. Riedl 等 ${ }^{[86]}$ 实现了 $\mathrm{H}$ 对 $\mathrm{SiC}$ 衬 底 $\mathrm{Si}$ 终端面上石墨烯的改性. $\mathrm{H}$ 原子饱和了祄底表面 $\mathrm{Si}$ 层的悬挂键和与石墨烯的共价键, 使得祄底与石墨烯之 间去耦合. 通过 ARPES 观察, 石墨烯的能带结构中狄 拉克点重新出现. 多余 $\mathrm{H}$ 原子吸附在表面石墨烯之上, 可以达到轻微 $\mathrm{P}$ 型掺杂的效果. 但是 $\mathrm{H}$ 原子到达衬底和 石墨烯层之间的具体微观过程和如何长时间保存这种 结构仍需探究.

Cheng 等 ${ }^{[87]}$ 利用第一性原理的计算探究了 $\mathrm{F}$ 原子的 改性作用. 通过对形成能的分析, $\mathrm{F}$ 的改性可分为三步 进行. 首先, $\mathrm{F}$ 插入到衬底和石墨烯层之间, 从而导致了 狄拉克点的产生. 之后, $\mathrm{F}$ 原子饱和祄底表面 $\mathrm{Si}$ 原子的 悬挂键, 即钝化作用, 达到了 $\mathrm{P}$ 型掺杂的效果. 最后, $\mathrm{F}$ 通过和表面石墨烯较为强烈的化学成键作用, 即表面修 饰作用, 加强了 $\mathrm{P}$ 型掺杂的效应. $\mathrm{F}$ 相较于 $\mathrm{H}$, 由于其很 强的电负性, 因此在进行表面修饰时, 会使能带打开. 在其他石墨烯体系中, $\mathrm{F}$ 已经被认为是可以良好的打开 能带的改性材料 ${ }^{[88,89]}$.

\section{3 分子对 $\mathrm{SiC}$ 衬底上石墨烯的改性}

由于分子比原子大，分子多附着在石墨烯表面，以 电荷转移的方式来达到 $\mathrm{P}$ 型掺杂和能带打开的效果. 分子与石墨烯相互作用需要有足够的强度, 才能进行改 性, 因此分子的功函数、电负性是需考虑的重要因 素 ${ }^{[71,90]} \cdot \mathrm{C}_{60}$ 没有足够强的吸引电子的能力 ${ }^{[91]}$, 因此无法 达到 P 型掺杂的效应. 能带的打开则依靠分子与石墨烯 中 $\mathrm{C}$ 原子成键, 使之从 $\mathrm{sp}^{2}$ 转变成 $\mathrm{sp}^{3}$ 的杂化方式来实现. Pinto 等 ${ }^{[92]}$ 通过将 F4-TCNQ 附着在石墨烯表面实现了对 石墨烯进行 P 型掺杂的效果. 如图 $10 \mathrm{a}$ 为其示意图. 当 增加 F4-TCNQ 的厚度时, 其功函数相应增加, 对电子 的束缚作用增强, 因此更容易产生 $\mathrm{P}$ 型掺杂的效应. 并 且此种结构在室温下是稳定的. Jee 等 ${ }^{[93]}$ 指出并五苯可 以作为保护层, 保证石墨烯独特的输运性质. 并五苯层 可以吸附于石墨烯层之上，和石墨烯之间没有强的作 用，并且可以轻易的移除，因此可以用有机分子来保护 制备的石墨烯层. Zhou 等 ${ }^{[94]}$ 通过在 $\mathrm{SiC}$ 衬底上石墨烯的 表面附着 $\mathrm{NO}_{2}$, 使石墨烯的能带打开带隙. STM 观察发 现 $\mathrm{NO}_{2}$ 多吸附于石墨烯表面, 随着附着分子数的增加, $\mathrm{P}$ 型掺杂程度增加. 图 $10 \mathrm{~b}$ 中显示了被 $\mathrm{NO}_{2}$ 表面修饰的 $\mathrm{C}$ 

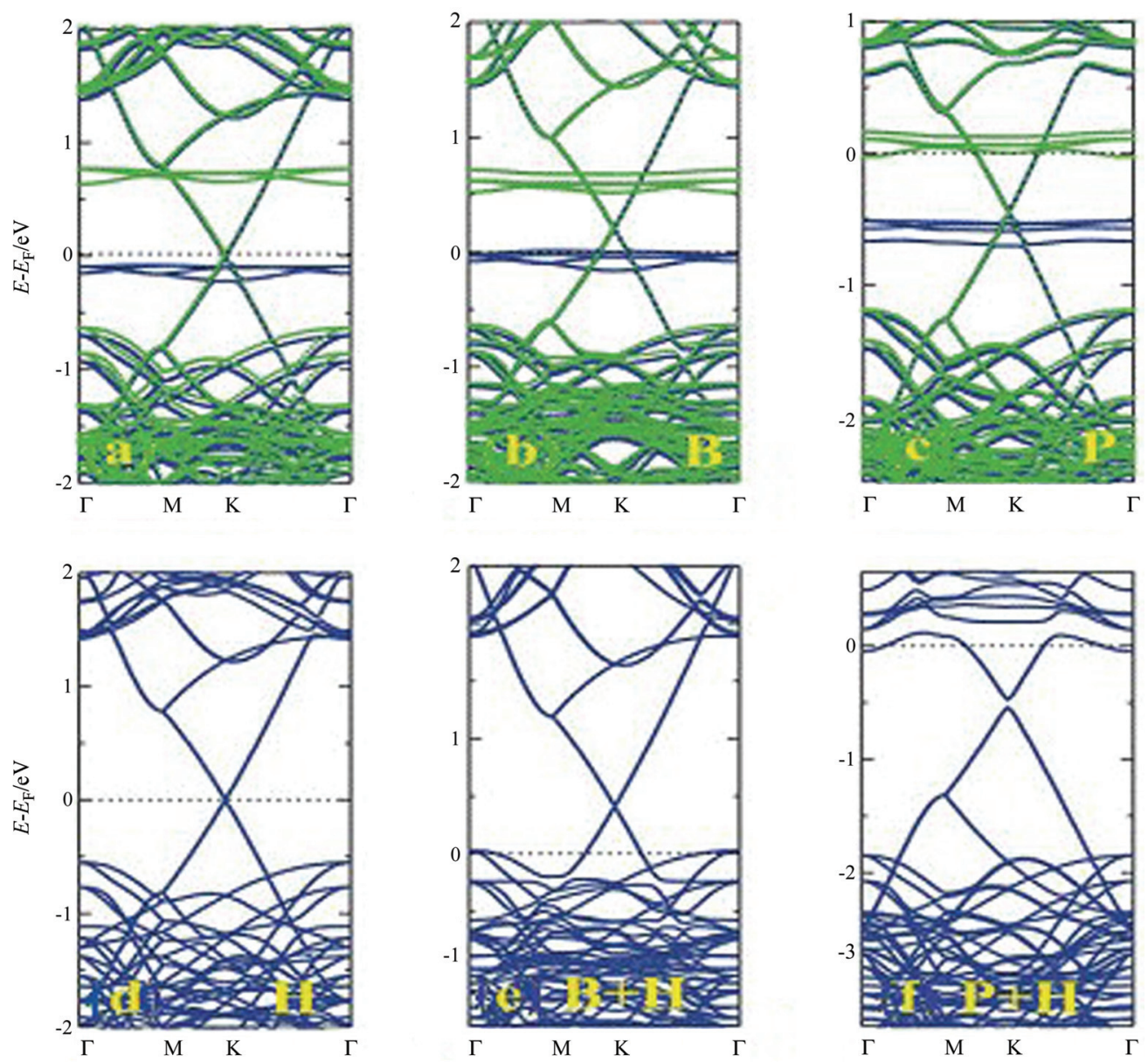

图 $9 \mathrm{SiC}$ 衬底 $\mathrm{C}$ 终端面上的石墨烯能带图. (a) 是未掺杂情况; (b)和(c)分别是在缓冲层掺杂 $\mathrm{B}$ 元素和 $\mathrm{P}$ 元素的能带图; (d $\sim f)$ 同 $(a \sim c)$ 类似, 区别 是悬挂键被 $\mathrm{H}$ 饱和与否 $\left(\mathrm{a}, \mathrm{b}, \mathrm{c}\right.$ 为未被 $\mathrm{H}$ 饱和的情况). 深蓝色线和浅绿色线分别代表了自旋向上和自旋向下的状态 ${ }^{[68]}$

Figure 9 The electronic band structures of the graphene-SiC system without doping (a) and doped by (b) B and (c) P in the buffer layer. Panels d f are the same as panels $\mathrm{a} \sim \mathrm{c}$ but the $\mathrm{C}$ atoms with dangling bonds at the $\mathrm{SiC}$ surface are passivated by $\mathrm{H}$ atoms. The blue/dark gray solid line and green/light gray dotted line represent the spin-up and spin-down states, respectively. The Fermi level is set at zero energy ${ }^{[68]}$

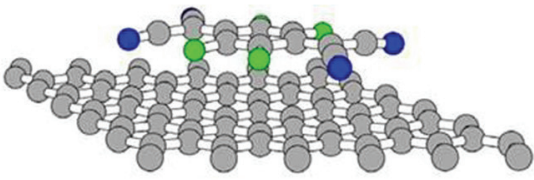

a

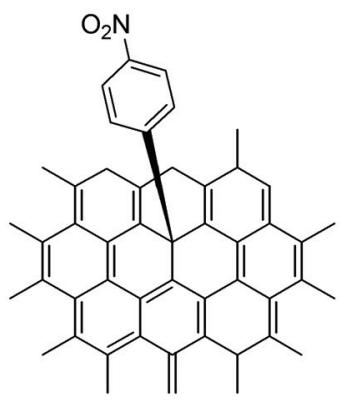

b

图 10 (a) F4-TCNQ 附着在石墨烯表面对石墨烯进行改性的模型 ${ }^{[92]}$; (b) 为将 $\mathrm{NO}_{2}$ 引入的示意图 ${ }^{[96]}$

Figure 10 (a) Molecule of F4-TCNQ on top of graphene ${ }^{[92]}$; (b) Schematic illustration of the spontaneous grafting of aryl groups to epitaxial graphene ${ }^{[96]}$

原子杂化成 $\mathrm{sp}^{3}$ 的成键方式 ${ }^{[96]}$. Lara-Avila 等 ${ }^{[95]}$ 通过实验 证实了利用聚合物产生的异质结构进行封装能够很好 地保持石墨烯层电子的电导特性, 而不像一般的化学改 性方法会降低电子的迁移率.

\section{$5 \mathrm{SiC}$ 衬底上石墨烯的应用}

石墨烯具有优异的电学光学特性, 因此在微纳电子
器件、储存技术、探测技术方面都有巨大应用潜力. $\mathrm{SiC}$ 祄底上外延石墨烯主要应用在器件方面, 如场效应管 等. 通过移植传统微电子工艺, 全 $\mathrm{C}$ 型的微纳器件已被 制造出来, 并已开展大量研究.

\section{$5.1 \mathrm{SiC}$ 衬底上石墨烯在器件方面的应用}

$\mathrm{SiC}$ 衬底上外延石墨烯在器件方面的应用处于初步 阶段. 外延石墨烯能隙较小，且传统的集成电路工艺在 
纳米量级实现有一定难度, 刻蚀等工艺会增加材料的杂 质散射，降低载流子的迁移率，因此一定程度上限制了 外延石墨烯在器件方面的发展. 为了实现外延石墨烯在 器件方面的大规模应用, 如何利用辅助材料及新型结构 来提高石墨烯品质如载流子迁移率, 提高导通关断电流 之比，减小损耗、响应时间是当前研究的热点 ${ }^{[70]}$.

\section{1 .1 基于 $\mathrm{SiC}$ 衬底上石, 墨烯器件的特性}

石墨烯由于其低的接触电阻、高的载流子迁移率、 小的温度系数、较强的抗腐蚀性等优异特性, 成为制造 场效应管、晶体管的理想材料 ${ }^{[97]}$. 制造的器件具有开通 电压低、发射电流密度高、发射稳定性好、使用寿命长 等优势. 碳纳米管同样在器件方面表现出优异的电学特 性, 但外延石墨烯具有碳纳米管所不具备的一些优势. 早在 2004 年, Berger 等 ${ }^{[19]}$ 通过将外延石墨烯和碳纳米管 的电学特性进行对比, 认为外延石墨烯是制造微纳器件 的理想材料. 首先, 在现有工艺条件下, 将已经定型的 碳纳米管很好地转移并组装到新的衬底上非常困难. 现 阶段难以通过改进转移工艺消除其对器件性能的不利 影响. 而外延石墨烯可直接利用其 $\mathrm{SiC}$ 衬底, 不需要进 行祄底转移. 其次, 向碳纳米管注入电子会导致很明显 的接触发热，这和碳纳米管与金属接触时的自然特性有 关. 由于 $\mathrm{C}$ 重构后与祄底良好的匹配性, 外延石墨烯的 这种发热现象并不明显 ${ }^{[98,99]}$. 因此外延法制备的石墨烯 在器件应用方面有其独特的优越性 ${ }^{[20]}$.

图 11c 显示了 $\mathrm{SiC}$ 片上高集成度的石墨烯纳器件, 通过改变栅极电压可以控制其导通和关断 ${ }^{[100 ~ 102]}$. 2008 年, Kedzierski 等 ${ }^{[103]}$ 通过将传统微电子工艺应用于外延 石墨烯，制造出基于石墨烯的场效应管. 2009 年, Guo 等 ${ }^{[104]}$ 通过第一性原理的计算, 研究了栅极电压对载流 子的影响, 验证了栅极电压对载流子有很好的调控作
用, 从而可控制器件的开通与关断. 2010 年, $\mathrm{Li}$ 等 ${ }^{[70]}$ 制 造出祄底表面层不同的场效应器件, 分析了 $\mathrm{SiC}$ 衬底上 石墨烯器件的特性. 祄底表面层不同, 石墨烯器件所拥 有的电学性质不同. 由于 $\mathrm{Si}$ 终端面上石墨烯有明显能 隙, 因此漏电流较小, 如图 $11 \mathrm{a}, \mathrm{b}$ 显示, 当门级偏置电 压 $V_{\mathrm{g}}=-3.5 \mathrm{~V}$ 时, 电导率最小, 导通关断电流之比可 以达到 30. 在 C 终端面上外延石墨烯中, $V_{\mathrm{g}}=0 \mathrm{~V}$ 时, 电 导率最小，其场效应管的极性难以分辨. 由于多层石墨 烯的屏蔽作用, 寄生电容效应较为严重, 并且导通关断 电流之比太小，导致漏电流太大，损耗严重. 因此虽然 $\mathrm{C}$ 终端面上石墨烯载流子迁移率比 $\mathrm{Si}$ 面情况的大, 但是 关断特性不理想.

\subsubsection{SiC 补底上石, 墨烯器件的工艺改进}

2010 年, $\mathrm{Li}$ 等 ${ }^{[70]}$ 分别制造出了顶端栅极、侧面栅极 的场效应管. 具体的工艺为, 利用掩膜版的控制作用, 将 $\mathrm{Pd} / \mathrm{Au}$ 沉积在已生成石墨烯的 $\mathrm{SiC}$ 晶片上实现金半接 触. 随后利用 PMMA 与电子束对样品进行光刻, 通过氧 等离子, 刻蚀出石墨烯带用作导电沟道. 栅极结构则是 通过沉积 Al 层来实现. 同年，如图 12a, Sprinkle 等 ${ }^{[105]}$ 通过利用刻蚀、光刻工艺与控制生长晶相，实现了纳米 带的直接生长, 避免了传统工艺中纳米带的无序边界 性, 提高了器件中载流子的迁移率, 且明显提高了器件 的集成度. 他们实现了在 $0.24 \mathrm{~cm}^{2}$ 的 $\mathrm{SiC}$ 晶片上集成 10000 多个晶体管. 另外利用 $\mathrm{NO}_{2}$ 可对石墨烯进行吸附 改性, 使器件的关断特性得以改善, 导通关断电流之比 达到 10 .

2011 年, Xue 等 ${ }^{[106]}$ 提出了一种新型结构来减弱衬 底对于石墨烯迁移率的影响. 他们通过在石墨烯与祄底 之间引入空气隔层, 并采用六方氮化硼这类在平面结构 下悬挂键与缺陷较少的材料作为栅极，从而减小了散射
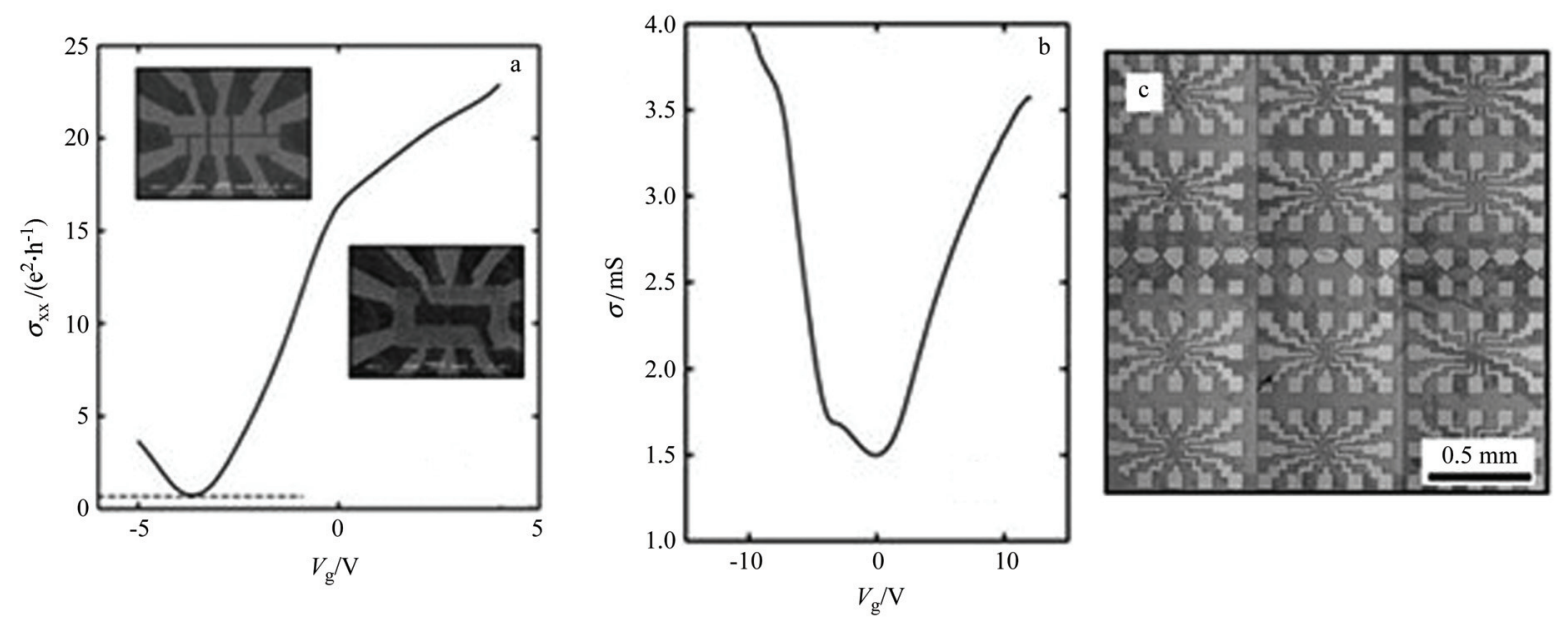

图 11 (a) $\mathrm{Si}$ 终端面上石墨烯电导率随门级偏置电压变化的关系, 插图是石墨烯带器件模型; (b)C 终端面上石墨烯电导率随栅极偏置电压变化的 关系; (c) $\mathrm{SiC}$ 片上由数百个石墨烯纳器件构成的集成结构 ${ }^{[70]}$

Figure 11 (a) Conductivity $\sigma$ is a function of gate voltage at $300 \mathrm{~K}$ for a single graphene layer on Si-face $\mathrm{SiC}$, Inset: the measured top gated ribbon, before and after gate deposition; (b) Conductivity as a function of gate voltage for a C-face multilayer epitaxial graphene Hall bar; (c) Example of integrated structures on a $\mathrm{SiC}$ chip, featuring a pattern of evaporated gold pads connecting a hundred micrometre and sub-micrometre size graphene ribbons (not seen) grown on the $\mathrm{C}$-face of $4 \mathrm{H}-\mathrm{SiC}$. The background contrast is an artefact from the tape on the back of the transparent $\mathrm{SiC}$ chip ${ }^{[70]}$ 

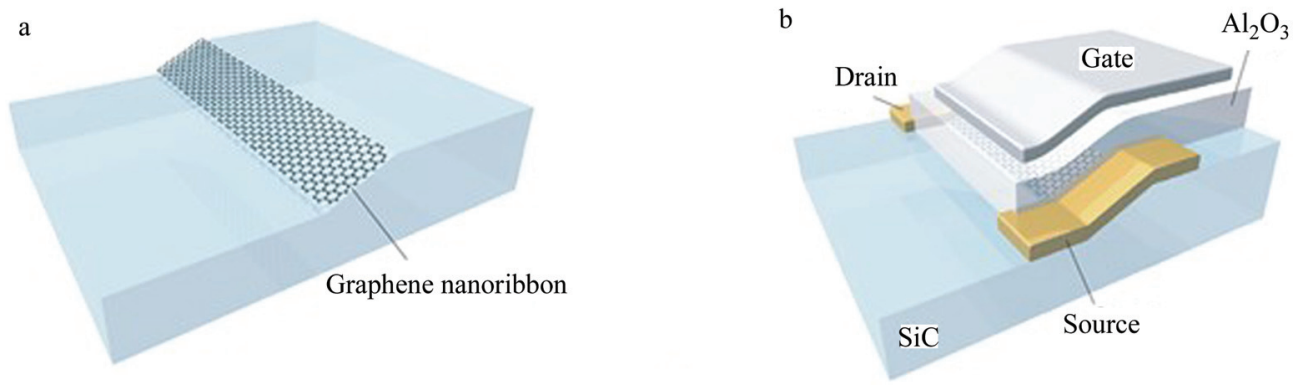

图 12 (a) 石墨烯在 $\mathrm{SiC}(110 n)$ 斜坡面生长示意图; (b) 具备源极、漏极接触, 石墨烯纳米带导电沟道, $\mathrm{Al}_{2} \mathrm{O}_{3}$ 绝缘介质层以及金属栅极的完整器件 示意图 ${ }^{[105]}$

Figure 12 (a) The image of graphene grown on a $\mathrm{SiC}(1 \overline{10} \mathrm{n})$ facet; (b) Complete device with source and drain contacts, graphene nanoribbon channel. $\mathrm{Al}_{2} \mathrm{O}_{3}$ gate dielectric and metal top-gate ${ }^{[105]}$

的影响. 2012 年, Hui 等 ${ }^{[107]}$ 通过控制氧化锌在石墨烯上 的沉积程度制造出不同掺杂效应的石墨烯场效应器件. 他们指出氧化锌的厚度直接决定了石墨烯的掺杂程度, 厚度越小, 则 $\mathrm{N}$ 型掺杂程度越高.

\section{$5.2 \mathrm{SiC}$ 衬底上石墨烯的其他应用}

石墨烯具有良好的可调控性. 依据不同应用所需的 电阻率和透明度等性质的不同, 不同品质的石墨烯有着 不同的应用.

外延石墨烯由于其在磁学方面优异的特性, 因此在 高密度储存和磁性传感器方面有光明的应用前景. 磁阻 由多层石墨烯之间的不一致性引起. Friedman 等 ${ }^{[65]}$ 在室 温下观察到了外延石墨烯的线性磁阻效应, 且磁阻很 高. 他们指出, 通过控制石墨烯生长的晶粒尺寸, 增大 外延石墨烯的不一致性, 可提高磁阻. Zhu 等 ${ }^{[108]}$ 研究了 不同尺寸石墨烯片层的磁性能. 他们通过拉曼光谱等实 验手段, 归纳出较小的石墨烯片层有较好的磁学性能.

纳米探测器的最终目标是实现单个原子或分子的 测量. 传统的探测器, 如固态气体探测器很难实现这一 目标, 因为其热效应产生的电子涨落和缺陷散射难以避 免, 从而产生了本征噪声信号. 而石墨烯具有稳定的化 学结构, 载流子和缺陷难以通过热效应产生. 当石墨烯 应用于探针时, 气体分子吸附在石墨烯表面而造成了局 部载流子浓度的变化, 载流子的变化以单个电子为量 级, 从而导致量子化的电阻变化. 因此石墨烯可以用作 敏感探针的制造 ${ }^{[109]}$. 外延石墨烯通过电氧化处理以后, 会在表面产生陷阴，这样的结构在光照下会释放载流 子, 因此延长了光生载流子的寿命. 所以将此法处理过 的石墨烯用于紫外线探测是可行的 ${ }^{[10]}$.

\section{6 结束语}

近年来, 利用 $\mathrm{SiC}$ 祄底外延法制备石墨烯的研究已 取得重要进展, 尤其在电子器件领域, 获得了广泛的关 注. 外延石墨烯的研究与应用关键之一是制备面积更 大、层数可控、缺陷更少的石墨烯. 在制备方面已有大 量的实验工作, 但为了进一步指导改善外延石墨烯的品 质, 加深对外延石墨烯的 $\mathrm{Si}$ 原子升华、 $\mathrm{C}$ 原子重构等生
长机理的认识, 需要结合多种研究方法(如蒙特卡洛、分 子动力学模拟等), 全面系统地考虑实验环境因素对外 延石墨烯的影响. 在改性方面, 理论计算已有一定工作, 主要是利用密度泛函理论对影响器件性能的因素进行 了分析，如能带打开程度、掺杂程度等. 在理论指导下， 结合当前的纳米工艺水平，系统深入有目的地去改性则 是今后的研究方向.

$\mathrm{SiC}$ 祄底上石墨烯基纳器件的制备测量已开展了一 定研究. 由于石墨烯是典型的纳米材料, 传统微电子工 艺不能很好的直接应用于其中(如刻蚀工艺会显著的降 低石墨烯的载流子迁移率等). 器件性能与理论计算预 测仍有一定差距，利用表面修饰进行可控改性仍需进一 步探索. 如何可控地改善器件性能、提高器件的集成度, 以满足大规模应用的需要是未来研究的重点.

\section{作者简介}

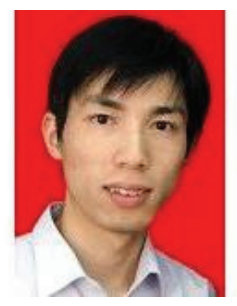

缪灵, 博士，讲师，硕士生导师。华中科技大学光学与电 子信息学院. 主要研究方向为石墨烯材料物理性质的第一性 原理计算及其在纳电子器件、电化学电容器、储氢等领域的 应用.

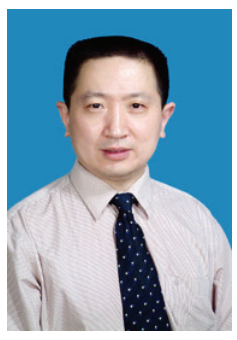

江建军, 博士, 教授, 博士生导师. 华中科技大学光学与 电子信息学院. 主要从事智能电子学及碳纳米材料的性质、应 用研究. 
方楠, 刘风, 刘小瑞, 廖瑞娴系华中科技大学光学与电子 信息学院 2009 级本科生, 参与课题 “不同祄底上石, 墨烯性质 及石墨烯器件的研究”.

\section{References}

[1] Geim, A. K.; Novoselov, K. S. Nat. Mater. 2007, 6, 183.

[2] Novoselov, K.; Morozov, S.; Mohinddin, T.; Ponomarenko, L.; Elias, D.; Yang, R.; Barbolina, I.; Blake, P.; Booth, T.; Jiang, D. Phys. Status Solidi B 2007, 244, 4106.

[3] Novoselov, K.; Geim, A.; Morozov, S.; Jiang, D.; Grigorieva, M. I. K. I. V.; Dubonos, S.; Firsov, A. Nature 2005, 438, 197.

[4] Zhang, Y.; Tan, Y. W.; Stormer, H. L.; Kim, P. Nature 2005, 438, 201.

[5] Novoselov, K. S.; Jiang, Z.; Zhang, Y.; Morozov, S.; Stormer, H.; Zeitler, U.; Maan, J.; Boebinger, G.; Kim, P.; Geim, A. Science 2007, $315,1379$.

[6] Ramesh, P.; Itkis, M. E.; Bekyarova, E.; Wang, F.; Niyogi, S.; Chi, X.; Berger, C.; de Heer, W.; Haddon, R. C. J. Am. Chem. Soc. 2010, $132,14429$.

[7] De Heer, W. A. Phys. Scripta 2012, 2012, 014004

[8] Novoselov, K.; Jiang, D.; Schedin, F.; Booth, T.; Khotkevich, V.; Morozov, S.; Geim, A. Proc. Natl. Acad. Sci. U. S. A. 2005, 102, 10451.

[9] De, S.; King, P. J.; Lotya, M.; O'Neill, A.; Doherty, E. M.; Hernandez, Y.; Duesberg, G. S.; Coleman, J. N. Small 2010, 6, 458.

[10] Koh, A. T. T.; Foong, Y. M.; Chua, D. H. C. Diam. Relat. Mater. 2012, 25, 98 .

[11] Wang, L.; Tian, L. H.; Wei, G. D.; Gao, F. M.; Zheng, J. J.; Yang, W. Y. J. Inorg. Mater. 2011, 26, 1009. (王霖, 田林海, 尉国栋, 高凤 梅, 郑金桔, 杨为佑, 无机材料学报, 2011, 26, 1009.)

[12] Li, X.; Cai, W.; An, J.; Kim, S.; Nah, J.; Yang, D.; Piner, R.; Velamakanni, A.; Jung, I.; Tutuc, E. Science 2009, 324, 1312.

[13] Dai, G. P.; Cooke, P. H.; Deng, S. Chem. Phys. Lett. 2012, 536, 113.

[14] Sutter, P. W.; Flege, J. I.; Sutter, E. A. Nat. Mater. 2008, 7, 406.

[15] Kim, E. S.; Shin, H. J.; Yoon, S. M.; Han, G. H.; Chae, S. J.; Bae, J. J.; Gunes, F.; Choi, J. Y.; Lee, Y. H. Appl. Phys. Lett. 2011, 99, 223102 .

[16] Van Bommel, A.; Crombeen, J.; Van Tooren, A. Surf. Sci. 1975, 48, 463.

[17] Owman, F.; Mårtensson, P. Surf. Sci. 1995, 330, L639.

[18] Van Elsbergen, V.; Kampen, T.; Mönch, W. Surf. Sci. 1996, 365, 443.

[19] Berger, C.; Song, Z.; Li, T.; Li, X.; Ogbazghi, A. Y.; Feng, R.; Dai, Z.; Marchenkov, A. N.; Conrad, E. H.; Phillip, N. J. Phys. Chem. B 2004, 108, 19912.

[20] De Heer, W. A.; Berger, C.; Wu, X.; First, P. N.; Conrad, E. H.; Li, X.; Li, T.; Sprinkle, M.; Hass, J.; Sadowski, M. L. Solid State Commun. 2007, 143, 92 .

[21] Berger, C.; Song, Z.; Li, X.; Wu, X.; Brown, N.; Naud, C.; Mayou, D.; Li, T.; Hass, J.; Marchenkov, A. N. Science 2006, 312, 1191.

[22] Sutter, P. Nat. Mater. 2009, 8, 171.

[23] Hupalo, M.; Conrad, E.; Tringides, M. Phys. Rev. B 2009, 80, 041401 .

[24] Bolen, M.; Harrison, S. E.; Biedermann, L.; Capano, M. A. J. Electron. Mater. 2009, 39, 2696.

[25] Norimatsu, W.; Takada, J.; Kusunoki, M. Phys. Rev. B 2011, 84, 035424.

[26] De Heer, W. A.; Berger, C.; Ruan, M.; Sprinkle, M.; Li, X.; Hu, Y.; Zhang, B.; Hankinson, J.; Conrad, E. Proc. Natl. Acad. Sci. U. S. A. 2011, 108,16900 .

[27] Daas, B. K.; Omar, S. U.; Shetu, S.; Daniels, K. M.; Ma, S.; Sudarshan, T. S.; Chandrashekhar, M. V. S. Cryst. Growth Des. 2012, 12, 3379 .

[28] Srivastava, N.; He, G.; Mende, P.; Feenstra, R.; Sun, Y. J. Phys. D: Appl. Phys. 2011, 45, 154001.

[29] Prakash, G.; Capano, M. A.; Bolen, M. L.; Zemlyanov, D.; Reifenberger, R. G. Carbon 2010, 48, 2383.

[30] Vecchio, C.; Sonde, S.; Bongiorno, C.; Rambach, M.; Yakimova, R.; Raineri, V.; Giannazzo, F. Nanoscale Res. Lett. 2011, 6, 1.

[31] Hass, J.; Millán-Otoya, J. E.; First, P. N.; Conrad, E. H. Phys. Rev. B 2008, 78, 205424.

[32] Lauffer, P.; Emtsev, K.; Graupner, R.; Seyller, T.; Ley, L.; Reshanov, S.; Weber, H. Phys. Rev. B 2008, 77, 155426

[33] Varchon, F.; Mallet, P.; Veuillen, J. Y.; Magaud, L. Phys. Rev. B
2008, 77, 235412

[34] Hite, J. K.; Twigg, M. E.; Tedesco, J. L.; Friedman, A. L.; Myers-Ward, R. L.; Eddy Jr., C. R.; Gaskill, D. K. Nano Lett. 2011, 11,1190 .

[35] Ohta, T.; Bartelt, N. C.; Nie, S.; Thürmer, K.; Kellogg, G. Phys. Rev. $B$ 2010, 81, 121411 .

[36] Ferrari, A.; Meyer, J.; Scardaci, V.; Casiraghi, C.; Lazzeri, M.; Mauri, F.; Piscanec, S.; Jiang, D.; Novoselov, K.; Roth, S. Phys. Rev. Lett. 2006, 97, 187401.

[37] Riedl, C.; Starke, U.; Bernhardt, J.; Franke, M.; Heinz, K. Phys. Rev. $B$ 2007, 76, 245406.

[38] Magaud, L.; Hiebel, F.; Varchon, F.; Mallet, P.; Veuillen, J. Y. Phys. Status Solidi-R 2009, 3, 172.

[39] Berger, C.; Wu, X.; First, P.; Conrad, E.; Li, X.; Sprinkle, M.; Hass, J.; Varchon, F.; Magaud, L.; Sadowski, M. Adv. Solid State Phys. 2008, 47, 145.

[40] Mathieu, C.; Barrett, N.; Rault, J.; Mi, Y.; Zhang, B.; de Heer, W.; Berger, C.; Conrad, E.; Renault, O. Phys. Rev. B 2011, 83, 235436.

[41] Emtsev, K.; Speck, F.; Seyller, T.; Ley, L.; Riley, J. Phys. Rev. B 2008, 77, 155303 .

[42] Varchon, F.; Feng, R.; Hass, J.; Li, X.; Nguyen, B. N.; Naud, C.; Mallet, P.; Veuillen, J. Y.; Berger, C.; Conrad, E. Phys. Rev. Lett. 2007, 99, 126805.

[43] Mattausch, A.; Pankratov, O. Phys. Rev. Lett. 2007, 99, 76802.

[44] Hass, J.; Varchon, F.; Millan-Otoya, J. E.; Sprinkle, M.; Sharma, N.; de Heer, W. A.; Berger, C.; First, P. N.; Magaud, L.; Conrad, E. H. Phys. Rev. Lett. 2008, 100, 125504.

[45] Hibino, H.; Tanabe, S.; Mizuno, S.; Kageshima, H. J. Phys. D: Appl. Phys. 2012, 45, 154008.

[46] Wu, X.; Hu, Y.; Ruan, M.; Madiomanana, N. K.; Hankinson, J.; Sprinkle, M.; Berger, C.; De Heer, W. A. Appl. Phys. Lett. 2009, 95, 223108 .

[47] Tang, J.; Liu, Z. L.; Kang, Z. Y.; Yan, W. S.; Xu, P. S.; Pan, H. B.; Wei, S. Q.; Gao, Y. Q.; Xu, X. G. Acta Phys. Chim. Sin. 2010, 26, 253. (唐军, 刘忠良, 康朝阳, 闵文盛, 徐彭寿, 潘海斌, 韦世强, 高玉强, 徐现刚, 物理化学学报, 2010, 26, 253.)

[48] Hass, J.; Feng, R.; Li, T.; Li, X.; Zong, Z.; De Heer, W.; First, P.; Conrad, E.; Jeffrey, C.; Berger, C. Appl. Phys. Lett. 2006, 89, 143106.

[49] Winnerl, S.; Orlita, M.; Plochocka, P.; Kossacki, P.; Potemski, M.; Winzer, T.; Malic, E.; Knorr, A.; Sprinkle, M.; Berger, C. Phys. Rev. Lett. 2011, 107, 237401.

[50] Faugeras, C.; Amado, M.; Kossacki, P.; Orlita, M.; Sprinkle, M.; Berger, C.; De Heer, W.; Potemski, M. Phys. Rev. Lett. 2009, 103, 186803.

[51] Plochocka, P.; Kossacki, P.; Golnik, A.; Kazimierczuk, T.; Berger, C.; De Heer, W.; Potemski, M. Phys. Rev. B 2009, 80, 245415.

[52] Hicks, J.; Sprinkle, M.; Shepperd, K.; Wang, F.; Tejeda, A.; Taleb-Ibrahimi, A.; Bertran, F.; Le Fèvre, P.; de Heer, W.; Berger, C. Phys. Rev. B 2011, 83, 205403.

[53] Sun, D.; Rioux, J.; Sipe, J.; Zou, Y.; Mihnev, M.; Berger, C.; de Heer, W. A.; First, P. N.; Norris, T. B. Phys. Rev. B 2011, 85, 165427.

[54] Faugeras, C.; Nerrière, A.; Potemski, M.; Mahmood, A.; Dujardin, E.; Berger, C.; De Heer, W. Appl. Phys. Lett. 2008, 92, 011914.

[55] First, P. N.; De Heer, W. A.; Seyller, T.; Berger, C.; Stroscio, J. A.; Moon, J. S. MRS Bull. 2010, 35, 296.

[56] Sclauzero, G.; Pasquarello, A. Phys. Rev. B 2012, 85, 161405.

[57] Ni, Z.; Chen, W.; Fan, X.; Kuo, J.; Yu, T.; Wee, A.; Shen, Z. Phys. Rev. B 2008, 77, 115416.

[58] Biedermann, L. B.; Bolen, M. L.; Capano, M. A.; Zemlyanov, D.; Reifenberger, R. Birck and NCN Publications. 2009, 79, 355.

[59] Hibino, H.; Mizuno, S.; Kageshima, H.; Nagase, M.; Yamaguchi, H. Phys. Rev. B 2009, 80, 085406.

[60] Starke, U.; Riedl, C. J. Phys. Condens. Matter 2009, 21, 134016.

[61] Hass, J.; De Heer, W.; Conrad, E. J. Phys: Condens. Matter 2008, 20, 323202 .

[62] Hiebel, F.; Mallet, P.; Varchon, F.; Magaud, L.; Veuillen, J. Phys. Rev. $B$ 2008, 78, 153412 .

[63] Wang, Z.; Irle, S.; Zheng, G.; Kusunoki, M.; Morokuma, K. J. Phys. Chem. C 2007, 111, 12960.

[64] Magaud, L.; Hiebel, F.; Varchon, F.; Mallet, P.; Veuillen, J. Y. Phys. Rev. B 2009, 79, 161405.

[65] Friedman, A. L.; Tedesco, J. L.; Campbell, P. M.; Culbertson, J. C.; Aifer, E.; Perkins, F. K.; Myers-Ward, R. L.; Hite, J. K.; Eddy Jr., C. 
R.; Jernigan, G. G. Nano Lett. 2010, 10, 3962.

[66] Sprinkle, M.; Soukiassian, P.; de Heer, W.; Berger, C.; Conrad, E. Phys. Status Solidi-R. 2009, 3, A91.

[67] Sun, D.; Divin, C.; Berger, C.; de Heer, W. A.; First, P. N.; Norris, T. B. Phys. Rev. Lett. 2010, 104, 136802.

[68] Huang, B.; Xiang, H.; Wei, S. H. Phys. Rev. B 2011, 83, 161405.

[69] Hu, Y. J; Jin, J.; Zhang, H.; Wu, P; Cai, C. X. Acta Phys-Chim. Sin. 2010, 26, 2073. (胡耀娟, 金娟, 张卉, 吴萍, 蔡称心, 物理化学学 报, 2010, 26, 2073.)

[70] Li, X.; Wu, X.; Sprinkle, M.; Ming, F.; Ruan, M.; Hu, Y.; Berger, C.; De Heer, W. A. Phys. Status Solidi A 2010, 207, 286.

[71] Bekyarova, E.; Sarkar, S.; Niyogi, S.; Itkis, M.; Haddon, R. J. Phys. D: Appl. Phys. 2012, 45, 154009.

[72] Choi, W.; Lahiri, I.; Seelaboyina, R.; Kang, Y. S. Crit. Rev. Solid. State 2010, 35, 52 .

[73] Tian, Y.; Zhao, Q. Y.; Hu, J.; Zhou, C.; Miao, L.; Jiang, J. J. Prog. Chem. 2012, 24, 512. (田圆, 赵倩莹, 胡靖, 周辰, 缪灵, 江建军, 化学进展, 2012, 24, 512.)

[74] Giovannetti, G.; Khomyakov, P.; Brocks, G.; Karpan, V.; Van den Brink, J.; Kelly, P. Phys. Rev. Lett. 2008, 101, 26803.

[75] Gierz, I.; Riedl, C.; Starke, U.; Ast, C. R.; Kern, K. Nano Lett. 2008, $8,4603$.

[76] Pletikosić, I.; Kralj, M.; Pervan, P.; Brako, R.; Coraux, J.; N’Diaye, A.; Busse, C.; Michely, T. Phys. Rev. Lett. 2009, 102, 56808.

[77] Preobrajenski, A.; Ng, M. L.; Vinogradov, A.; Mårtensson, N. Phys. Rev. B 2008, 78, 073401.

[78] Moritz, W.; Wang, B.; Bocquet, M. L.; Brugger, T.; Greber, T.; Wintterlin, J.; Günther, S. Phys. Rev. Lett. 2010, 104, 136102.

[79] Premlal, B.; Cranney, M.; Vonau, F.; Aubel, D.; Casterman, D.; De Souza, M.; Simon, L. Appl. Phys. Lett. 2009, 94, 263115.

[80] Varykhalov, A.; Sánchez-Barriga, J.; Shikin, A.; Biswas, C.; Vescovo, E.; Rybkin, A.; Marchenko, D.; Rader, O. Phys. Rev. Lett. 2008, 101, 157601 .

[81] Gierz, I.; Suzuki, T.; Weitz, R. T.; Lee, D. S.; Krauss, B.; Riedl, C.; Starke, U.; Höchst, H.; Smet, J. H.; Ast, C. R. Phys. Rev. B 2010, 81, 235408.

[82] Panchakarla, L.; Subrahmanyam, K.; Saha, S.; Govindaraj, A.; Krishnamurthy, H.; Waghmare, U.; Rao, C. Adv. Mater. 2009, 21, 4726.

[83] Gao, R. L.; Miao, L.; Song, J. Q.; Wu, Y. 2009 China Functional Materials Technology and Industry Forum, 2009 supplement. (高瑞 玲, 缪灵, 宋家琪, 吴忧, 2009 中国功能材料科技与产业高层论 坛论文集, 2009, 增刊.)

[84] Guisinger, N. P.; Rutter, G. M.; Crain, J. N.; First, P. N.; Stroscio, J. A. NanoLett. 2009, 9, 1462.

[85] Balog, R.; Jørgensen, B.; Wells, J.; Lægsgaard, E.; Hofmann, P.; Besenbacher, F.; Hornekær, L. J. Am. Chem. Soc. 2009, 131, 8744

[86] Riedl, C.; Coletti, C.; Iwasaki, T.; Zakharov, A.; Starke, U. Phys. Rev. Lett. 2009, 103, 246804.

[87] Cheng, Y.; Kaloni, T.; Huang, G.; Schwingenschlögl, U. Appl. Phys. Lett. 2011, 99, 053117.
[88] Khare, B. N.; Wilhite, P.; Meyyappan, M. Nanotechnology 2004, 15, 1650.

[89] Robinson, J. T.; Burgess, J. S.; Junkermeier, C. E.; Badescu, S. C.; Reinecke, T. L.; Perkins, F. K.; Zalalutdniov, M. K.; Baldwin, J. W.; Culbertson, J. C.; Sheehan, P. E. Nano Lett. 2010, 10, 3001.

[90] Niyogi, S.; Bekyarova, E.; Hong, J.; Khizroev, S.; Berger, C.; de Heer, W.; Haddon, R. C. J. Phys. Chem. Lett. 2011, 2, 2487.

[91] Sque, S.; Jones, R.; Goss, J.; Briddon, P.; Öberg, S. J. Phys. Condens. Matter 2005, 17, L21.

[92] Pinto, H.; Jones, R.; Goss, J.; Briddon, P. J. Phys: Condens. Matter. 2009, 21, 402001 .

[93] Jee, H.; Han, J. H.; Hwang, H. N.; Kim, B.; Kim, H.; Kim, Y. D.; Hwang, C. C. Appl. Phys. Lett. 2009, 95, 093107.

[94] Zhou, S.; Siegel, D.; Fedorov, A.; Lanzara, A. Phys. Rev. Lett. 2008 101, 86402.

[95] Lara-Avila, S.; Moth-Poulsen, K.; Yakimova, R.; Bjornholm, T.; Fal'ko, V.; Tzalenchuk, A.; Kubatkin, S. Adv. Mater. 2011, 23, 878.

[96] Bekyarova, E.; Itkis, M. E.; Ramesh, P.; Berger, C.; Sprinkle, M.; De Heer, W. A.; Haddon, R. C. J. Am. Chem. Soc. 2009, 131, 1336.

[97] Stolyarova, E.; Stolyarov, D.; Bolotin, K.; Ryu, S.; Liu, L.; Rim, K.; Klima, M.; Hybertsen, M.; Pogorelsky, I.; Pavlishin, I. Nano Lett. 2008, 9, 332.

[98] Bekyarova, E.; Itkis, M. E.; Ramesh, P.; Haddon, R. C. Phys. Status Solidi-R 2009, 3, 184.

[99] Emery, J. D.; Wang, Q. H.; Zarrouati, M.; Fenter, P.; Hersam, M. C.; Bedzyk, M. J. Surf. Sci. 2011, 605, 1685.

[100] Soin, N.; Roy, S. S.; Lim, T. H.; McLaughlin, J. A. D. Mater. Chem. Phys. 2011, 129, 1051.

[101] Novoselov, K.; Geim, A.; Morozov, S.; Jiang, D.; Zhang, Y.; Dubonos, S.; Grigorieva, I.; Firsov, A. Science 2004, 306, 666.

[102] Kopylov, S.; Tzalenchuk, A.; Kubatkin, S.; Fal'ko, V. I. Appl. Phys. Lett. 2010, 97, 112109.

[103] Kedzierski, J.; Hsu, P. L.; Healey, P.; Wyatt, P. W.; Keast, C. L.; Sprinkle, M.; Berger, C.; de Heer, W. A. IEEE T. Electron. Dev. 2008, 55, 2078

[104] Guo, Y.; Guo, W.; Chen, C. Phys. Rev. B 2009, 80, 085424.

[105] Sprinkle, M.; Ruan, M.; Hu, Y.; Hankinson, J.; Rubio-Roy, M.; Zhang, B.; Wu, X.; Berger, C.; De Heer, W. Nat. Nanotechnol. 2010, 5,727 .

[106] Xue, J.; Sanchez-Yamagishi, J.; Bulmash, D.; Jacquod, P.; Deshpande, A.; Watanabe, K.; Taniguchi, T.; Jarillo-Herrero, P.; LeRoy, B. J. Nat. Mater. 2011, 10, 282.

[107] Hui, Y. Y.; Tai, G.; Sun, Z.; Xu, Z.; Wang, N.; Yan, F.; Lau, S. P. Nanoscale 2012, 4, 3118.

[108] Zhu, J.; Luo, Z.; Wu, S.; Haldolaarachchige, N.; Young, D. P.; Wei, S.; Guo, Z. J. Mater. Chem. 2011, 22, 835.

[109] Schedin, F.; Geim, A.; Morozov, S.; Hill, E.; Blake, P.; Katsnelson, M.; Novoselov, K. Nat. Mater. 2007, 6, 652.

[110] Itkis, M. E.; Wang, F.; Ramesh, P.; Bekyarova, E.; Niyogi, S.; Chi, X.; Berger, C.; de Heer, W. A.; Haddon, R. C. Appl. Phys. Lett. 2011, 98, 093115.

(Cheng, B.; Fan, Y.) 